\title{
ON LOCALLY COMPACT GROUPS WITH INVARIANT MEANS
}

\author{
H. LEPTIN
}

In two recent papers [5], [6] I considered certain conditions for locally compact groups and I mentioned at the end of [6] that there might be close connections between these properties and the theory of groups with invariant means. The purpose of the present note is to study these relations. Among other things, I will prove that (with the notation of [6]) $I(G)=1$ implies that $G$ is amenable (i.e., has an invariant mean) and that amenability of $G$ is equivalent to $G$ being a $K_{2}$-group in the sense of [5]. Concerning the remark at the end of [6], M. Kneser pointed out to me that $I(G)>1$ implies $I(G)=\infty$. Indeed, it is almost immediate that

$$
\inf _{|U|>0} \frac{|A B U|}{|U|} \geqq \inf _{|U|>0} \frac{|A U|}{|U|} \inf _{|U|>0} \frac{|B U|}{|U|}
$$

for arbitrary compact sets $A$ and $B$ in $G$. The group $G=\operatorname{SL}(2, R)$ shows that the answer to the question in the second remark is "no": If $H$ is the solvable subgroup of triangular matrices in $G$, then $G \backslash H$ is compact, $I(H)=1$, but $I(G)=\infty$, because $G$ is not amenable.

I wish to thank Professors P. Eymard, F. P. Greenleaf and H. Reiter for helpful comments and for bringing to my attention a couple of relevant papers.

1. Let $G$ be a locally compact group with left invariant Haar measure $d x$. The Haar measure of a measurable subset $X$ of $G$ is denoted by $|X|$. We start with a series of conditions for $G$ :

(A) For every $\epsilon>0$ and every compact $K \subset G$ there exists a compact set $U \subset G$ such that $|K U|<(1+\epsilon)|U|$.

Using the invariant $I(G)$ introduced in [6], this means $I(G)=1$.

(B) $G$ is amenable.

This means that there exists a nontrivial left invariant mean on the Banach space of all complex valued, bounded, continuous functions on $G$. F. Greenleaf proved in [3] and [4], that this is equivalent to the existence of invariant means on any one of the following spaces: $L^{\infty}(G)=$ bounded measurable functions, $C_{r u}(G)=$ bounded right uniformly continuous functions, $C_{u}(G)=$ bounded two-sided uniformly continuous functions.

Received by the editors January 26, 1967. 
There are many other conditions all equivalent to (B) (for references see [3]):

(B $\left.{ }^{1}\right)$ Hulanicki's condition. All irreducible continuous unitary representations of $G$ are weakly contained in the left regular representation $\lambda$ of $G$ in $L^{2}(G)$.

This condition is already satisfied if the trivial one-dimensional representation is weakly contained in $\lambda$, which in turn is equivalent to

$\left(\mathrm{B}^{2}\right)$ The constant function 1 is, on every compact set of $G$, the uniform limit of positive definite functions of the form $\phi * \phi^{*}$ with continuous functions $\phi$ with compact carrier.

For a discussion of these and other properties of $G$ see, e.g., [2, $\S 18.3]$.

$\left(\mathrm{B}^{3}\right)$ Reiter's Condition. For every compact $K \subset G$ and every $\epsilon>0$ there exists a function $f \in L^{1}(G)$ with $f \geqq 0,|f|_{1}=1$ and $\left|{ }_{x} f-f\right|_{1}<\epsilon$ for all $x \in K$.

Here ${ }_{x} f$ is the function ${ }_{x} f(t)=f\left(x^{-1} t\right)$ and ||$_{1}$ is the norm in $L^{1}$.

$\left(\mathrm{B}^{4}\right)$ FIXED POINT PROPERTY. If $G$ acts affinely on a compact convex subset $S$ of a locally convex topological vector space, with the map $G \times S \rightarrow S$ continuous, then $S$ contains a fixed point under the action of $G$.

$\left(\mathrm{B}^{5}\right)$ F $\phi$ LNER's CONDITION. For every compact set $K \subset G$ and every $\epsilon>0$ there exists a compact set $U \subset G$ such that $|x U \triangle U|<\epsilon \cdot|U|$ for all $x \in K$.

( $X \triangle Y$ is the symmetric difference of the sets $X$ and $Y$.) Condition $\left(B^{5}\right)$ is easily seen to be equivalent to

(C) For every $K$ and $\epsilon$ as in $\left(\mathrm{B}^{5}\right)$ there exists a compact $U \subset G$ with $|U|>0$ such that $|U| /|x U \cap U|<1+\epsilon$ for all $x \in K$.

These (i.e., (B), $\left(\mathrm{B}^{1}\right)$ through $\left(\mathrm{B}^{5}\right)$ and $(\mathrm{C})$ ) are the conditions known all to be equivalent. Condition $(\mathrm{C})$ can be weakened to

$\left(C_{B}\right)$ There exists a constant $B>1$ such that for every compact $K \subset G$ there exists a compact $U \subset G$ with $|U|>0$ and $|U| /|x U \cap U|<B$ for all $x \in K$.

This is our condition (B) from [5, p. 113]. In [5] we defined $p$ admissible measures on $G$ as positive regular Borel measures $\mu$ such that $\mu * f \in L^{p}(G)$ for all $f \in L^{p}(G)$; in short, $\mu * L^{p} \subset L^{p}, p \geqq 1$. We proved that every $\left(C_{B}\right)$ implies

(D) For all $p \geqq 1$ every $p$-admissible measure on $G$ is bounded.

Let $p>1$ be fixed. Then (D) implies

$\left(\mathrm{D}_{\mathrm{p}}\right)$ Every $p$-admissible measure on $G$ is bounded.

For $\phi \in L^{1}$ let $\lambda_{p}(\phi)$ be the convolution operator on $L^{p}$, defined by $\lambda_{p}(\phi) f=\phi * f$, and $\left|\lambda_{p}(\phi)\right|$ its operator-norm. So $\lambda_{p}: \phi \rightarrow \lambda_{p}(\phi)$ is the left regular representation of $L^{1}$ in $L^{p}$. Let $\lambda_{2}=\lambda$. In [1] M. M. Day has a condition which, in the case of groups, is closely connected with 
(E) For every $p \geqq 1$ and all positive $\phi \in L^{1}$

$$
\left|\lambda_{p}(\phi)\right|=|\phi|_{1}=\int \phi d x .
$$

Again, this may be weakened to

$\left(\mathrm{E}_{\mathrm{p}}\right)$ For each positive $\phi \in L^{1}$ one has $\left|\lambda_{p}(\phi)\right|=|\phi|_{1}$.

2. Now we investigate the relations among the conditions (A) through $\left(E_{p}\right)$ listed above.

Proposition 1. (A) implies (B).

We prove $(\mathrm{A}) \Rightarrow(\mathrm{C})$. Let $\epsilon, K$ and $U$ be as in (C) and assume $e=e^{2} \in K$. For $x \in K$ we have $K U \supset x U \cup U$, hence $(1+\epsilon)|U| \geqq|K U|$ $\geqq|x U \cup U|=|x U|+|U|-|x U \cap U|=2|U|-|x U \cap U|$. Therefore $(1-\epsilon)|U| \leqq|x U \cap U|$. If $\epsilon<\frac{1}{2}$, this implies

$$
|U| /|x U \cap U|<1 /(1-\epsilon)<1+2 \epsilon .
$$

Proposition 2. The following schema of implications holds $(p>1)$ :

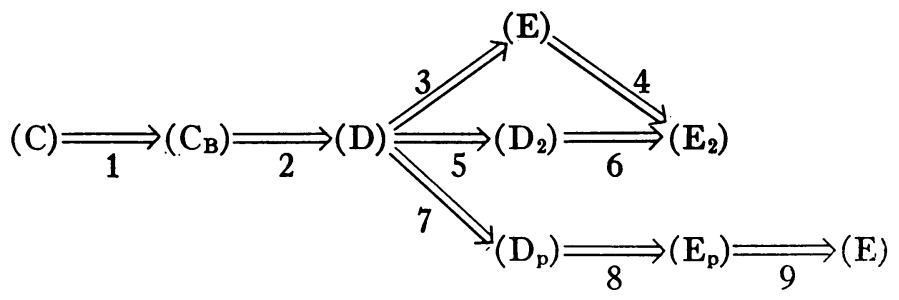

Proof. Implications 1, 4, 5 and 7 are trivial, 2 was proved in [5], 6 is a special case of 8 and 3 follows from 7 and 8 for all $p, 9$ is a known application of M. Riesz's convexity theorem; hence we need prove only 8.

To prove $\left(\mathrm{D}_{\mathrm{p}}\right) \Rightarrow\left(\mathrm{E}_{\mathrm{p}}\right)$ assume that $\left(\mathrm{E}_{\mathrm{p}}\right)$ does not hold for $G$. Then there exists $\phi \in L^{1}$ with $\phi \geqq 0, \int \phi d x=|\phi|_{1}=1$, but $\left|\lambda_{p}(\phi)\right|<1$. If $\phi^{n}$ is the $n$th power of $\phi$ in $L^{1}$, we have $\left|\phi^{n}\right|_{1}=|\phi|_{1}^{n}=1,\left|\sum_{i=1}^{n} \phi^{i}\right|_{1}$ $=\sum_{i}^{n}|\phi|_{1}^{i}=n$, because all functions are positive. Hence $\Phi=\sum_{1}^{\infty} \phi^{k}$ is a positive measurable function which is not in $L^{1}$ and so defines an unbounded measure $\Phi d x$ on $G$. Let us prove that $\Phi$ is locally in tegrable on $G$. Let $q=p /(p-1)$ and take positive functions $f \in L^{p}$ and $g \in L^{q}$ with $(f \mid g)=\int f g d x>1$. If $x_{0} \in K$ and $x_{0} f, x_{0} g$ are the left translates of $f$ and $g$ we have $\left(\left.{ }_{x_{0}} f\right|_{x_{0}} g\right)=(f \mid g)>1$ and because $x \rightarrow\left({ }_{x} f \mid{ }_{x_{0}} g\right)$ is continuous, $\left(\left.{ }_{x} f\right|_{x_{0}} g\right)>1$ for all $x$ in a neighborhood $U\left(x_{0}\right)$ of $x$. Let $x_{1}, x_{2}$, $\cdots, x_{r}$ be such that $K \subset \bigcup_{i=1}^{r} U\left(x_{i}\right)$ and define $h=\sum_{i=1}^{r} x_{i} g \in L^{q}$. Then it follows that for all $x \in K$ 


$$
\left({ }_{x} f \mid h\right) \geqq\left(\left.{ }_{x} f\right|_{x_{i} g} g\right)>1
$$

if $x \in U\left(x_{i}\right)$. Hence

$$
\begin{aligned}
\int_{K} \Phi d x & \leqq \int_{G} \Phi(x)\left({ }_{x} f \mid h\right) d x=\iint \Phi(x) f\left(x^{-1} y\right) h(y) d y d x \\
& =\int(\Phi * f)(y) h(y) d y=(\Phi * f \mid h)<\infty .
\end{aligned}
$$

So $\Phi d x$ is an unbounded Borel measure on $G$. On the other hand we have for every positive $f \in L^{p}$

$$
\begin{aligned}
|\Phi * f|_{p} & =\lim _{n \rightarrow \infty}\left|\sum_{1}^{n} \phi^{k} * f\right|_{p} \leqq \lim _{p \rightarrow \infty} \sum_{1}^{n}\left|\lambda_{p}(\phi)^{k} f\right|_{p} \\
& \leqq \lim _{n \rightarrow \infty} \sum_{1}^{n}\left|\lambda_{p}(\phi)\right|^{k}|f|_{p} \leqq \frac{\left|\lambda_{p}(\phi)\right|}{1-\left|\lambda_{p}(\phi)\right|} \cdot|f|_{p}
\end{aligned}
$$

consequently $\Phi d x$ defines a bounded linear transformation from $L^{p}$ in to itself. It follows that $\left(D_{p}\right)$ is violated.

The following proposition is the main step towards our final theorem:

Proposition 3. $\left(\mathrm{E}_{2}\right)$ implies $\left(\mathrm{B}^{1}\right)$.

Proof. We must show that the one-dimensional *-representation $\iota: \phi \rightarrow \iota(\phi)=\int \phi d x$ of $L^{1}$ (which corresponds to the identity representation $i(x)=1$ of $G$ ) can be factored by $\lambda$. Let $R$ be the $C^{*}$-algebra on $L^{2}(G)$ generated by $\lambda\left(L^{1}\right)=\Lambda$. Define $\kappa$ on $\Lambda$ by $\kappa(\lambda(\phi))=\iota(\phi)$. Then $\kappa$ is a $*$-representation of $\Lambda$ and our proof will be finished if we can prove that $\kappa$ is bounded in the operator norm of $R$.

If $\phi \in L^{1}$ is positive, then $\left(\mathrm{E}_{2}\right)$ yields

$$
|\lambda(\phi)|=|\phi|_{1}=\int \phi d x=\iota(\phi)=\kappa(\lambda(\phi)),
$$

hence if $z=x-y$ with $x=\lambda(\phi), y=\lambda(\psi), \phi$ and $\psi$ positive:

$$
|\kappa(z)|=|\kappa(x)-\kappa(y)|=|| x|-| y|| \leqq|x-y|=|z|,
$$

i.e., $\kappa$ is bounded by 1 on the real algebra of all $\lambda(\phi), \phi$ real in $L^{1}$. Let $i=(-1)^{1 / 2}$. For real $\phi$ and $\psi$ in $L^{1}$ we have

$$
\begin{aligned}
|\lambda(\phi-i \psi)| & =\sup _{|f|_{2}=1}|(\phi-i \psi) * f|_{2}=\sup _{|f|_{2}=1}|(\phi+i \psi) * \bar{f}|_{2} \\
& =\sup _{|\rho|_{2}=1}|(\phi+i \psi) * g|_{2}=|\lambda(\phi+i \psi)|
\end{aligned}
$$


where $\bar{f}$ is the conjugate complex function of $f$. Then with $x=\lambda(\phi)$, $y=\lambda(\psi), z=x+i y$ and $\bar{z}=x-i y$, we have

$$
\begin{aligned}
|\kappa z| & \leqq|\kappa x|+|\kappa y| \\
& \leqq|x|+|y|=\frac{1}{2}(|z+\bar{z}|+|z-\bar{z}|) \\
& \leqq \frac{1}{2}(|z|+|\bar{z}|+|z|+|\bar{z}|)=2|z|,
\end{aligned}
$$

therefore $\kappa$ is bounded by 2 and $\kappa$ can be extended to a continuous $*$-representation $\tilde{\kappa}$ of $R$. Because $\iota=\tilde{\kappa} \lambda$, Proposition 3 follows.

The main results of this paper follow from the last two propositions:

Theorem 1. The conditions (B), (C), $\left(\mathrm{C}_{\mathrm{B}}\right)$ (any $\left.\mathrm{B}>1\right),(\mathrm{D}),\left(\mathrm{D}_{p}\right)$, (E) and $\left(\mathrm{E}_{p}\right)$ are equivalent.

3. In general I do not know whether (B) implies (A) or not. But at least for many important cases this is true:

THEOREM 2. If $G$ contains an open normal subgroup $H$ which is almost connected (i.e., $H$ modulo the connected component of the identity is compact), then (B) implies (A).

Proof. Assume $G$ satisfies (B) and contains closed normal subgroups $H$ and $R$ such that $G / H$ is discrete, $H$ is almost connected and $R$ is the radical of $H$. From Corollary 2.2.3 in [3] it follows that $H / R$ is compact. Because $R$ is solvable we have $I(R)=1$, therefore Theorem 1 in [6] gives

$$
I(G) \leqq I(G / H) I(H / R) I(R)=I(G / H) .
$$

Hence it suffices to prove the theorem for a discrete group $G$. In this case we use condition (C). Let $K$ be finite in $G,|K|=n$, and $\epsilon>0$. There exists a finite set $U$ in $G$ such that

$$
|x U \cap U| /|U|>1-\epsilon / n \text { for all } x \in K .
$$

If we denote by $A-B$ the complement of $B$ in $A$, it follows that

$$
\begin{aligned}
\frac{|K U|}{|U|} & =\frac{\left|\bigcup_{x \in K} x U\right|}{|U|}=\frac{\mid U \cup\left\{{\underset{K}{K}}_{(x U-x U \cap U)\} \mid}\right.}{|U|} \\
& \leqq 1+\sum_{x \in K} \frac{|x U-x U \cap U|}{|U|} \leqq 1+n \frac{\epsilon}{n}=1+\epsilon .
\end{aligned}
$$

This means that (A) is satisfied and proves the theorem.

Added in Proof. Meanwhile Emerson and Greenleaf proved, that 
(B) implies (A) for arbitrary locally compact groups, see Math. Z. 102 (1967), 170-184.

\section{REFERENCES}

1. M. M. Day, Convolutions, means and spectra, Illinois J. Math. 8 (1964), 100-111.

2. J. Dixmier, Les $C^{*}$-algebras et leurs representations, Gauthier-Villars, Paris, 1964.

3. F. P. Greenleaf, Invariant means on locally compact groups and their applications, (to appear).

4. - Folner conditions for locally compact groups, (to appear).

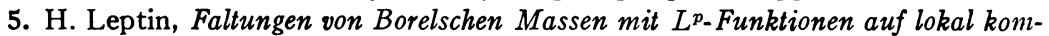
pakten Gruppen, Math. Ann. 163 (1966), 111-117.

6. - On a certain invariant of a locally compact group, Bull. Amer. Math. Soc. 72 (1966), 870-874.

Institut für Angewandte Mathematik, Universität Heidelberg, West Germany 